\section{Synovectomy of the Knee in Rheumatoid Arthritis}

Two inter-related studies have been made of the outcome of knee synovectomy. The first is concerned with the early postoperative period of recovery from the temporary impairment of function stemming directly from the surgical intervention. The second is concerned with the long-term outcome, with particular emphasis on changes in serial radiographs.

Part I. Short-term Observations. By A. V. CAMP (Stoke Mandeville Hospital).

Synovectomy of the knee for rheumatoid arthritis has been used as a form of treatment for many years. Little, however, is known of the best method of postoperative treatment and the long-term results. The success of the operation may be measured by the loss of pain and swelling in the knee, by the range of movement achieved, and by the strength of the quadriceps muscle.

A study has been made of all patients with rheumatoid arthritis admitted for knee synovectomy over a period of 2 years. The range of movement postoperatively and the quadriceps function have been measured and the operative results recorded. The factors governing the recovery of knee function have been studied in order to forecast the short-term prognosis in individual patients.

Part II. Long-Term Results. By A. R. TAYLOR and J. ScOTt HARBison (Stoke Mandeville Hospital).

94 knees were submitted to synovectomy and foilowed for periods of up to 6 years, special attention being paid to changes recorded in serial radiographs.

\section{Discussion}

DR. G. D. KERSLEY (Bath) The Bath series, which now dates back about 20 years, was first written up by Mr. London, and is being reassessed by Mr. Bliss, gave very much the same results. The fact which we learnt fairly early on, and which I think agrees with what we have just been told, is that you should tackle these cases before there is much radiological change. For a good many years, therefore, we have excluded patients in whom $x$ rays showed marked changes. The only point on which we might slightly disagree with you is that we think that at the end of a fortnight, in a very large number of cases, a manipulation, sometimes together with a steroid injection, expedites a good result.

DR. E. N. GLICK (London) I should like to know what happens in the unoperated knee.

MR. TAYLOR We do not have information about the progress of the disease in other joints.

DR. A. G. S. HILL (Stoke Mandeville) This question is being asked in the United Kingdom synovectomy trial: 'Did the patient have a general exacerbation and if so did the operated knee participate in it?'. I should have said, off the cuff, that in these synovectomy cases one sees both patterns: the synovectomized knee may remain cool amidst the fire of the general exacerbation, or, in contrast, it may share in the fire.
MR. KATES (London) When I started synovectomies of the knee 8 years ago I was an optimist, but my optimism has now diminished considerably. In my experience one cannot anticipate operative findings in the knee joint on $x$-ray findings alone. I have had a number of shocks, when $x$ rays have looked fairly normal but marked erosion of cartilage on both femoral and tibial condyles has been found at operation; in such cases my own feeling has been that the disease process was too far advanced for synovectomy to be successful although the $x$ rays showed little change. We have also seen that synovium regenerates completely within 6 to 12 months of surgery, and that even if one performs a complete anterior synovectomy of the knee, only 60 per cent. of the synovium can be removed. Histologically, the regenerated synovium resembles the original. I think that the only way to solve the problem whether synovectomy is of value or not, is by the United Kingdom Trials. Those of us who have been and are taking part in these trials have been told that there is a great shortage of knees, and yet here we have a series treated in the past 2 years which seems to have missed the net.

DR. A. G. S. HILl (Stoke Mandeville) The criteria for the Trial, as you know, Mr. Kates, are fairly strict, and I should have thought that only 10 or 15 per cent. of the cases that we submit to synovectomy are eligible for the Trial. For this there are all sorts of reasons (the erosions are more than minimal, the serology is negative, or some factor is present that excludes them from the Trial). Does that answer the query? Is this your experience, Mr. Kates?

MR. A. KATES (London) Yes. We find that we are able to send very few cases through to the Trial.

DR. A. ST. J. DIXON (Bath) This raises a point of fundamental importance in this Trial. If in fact from your experience only 15 per cent. are eligible, the conclusions that we draw from our Trial when we finish it will not be applicable to the majority of synovectomies.

DR. HILL I think this is a well-made point.

DR. K. N. LLOYD (Cardiff) Regarding the degree of joint damage before admitting these patients to synovectomy, we have heard much about the 'joint space'. I don't like this word at all because it gives the wrong impression. What we are talking about as 'joint space' is the thickness of the articular cartilage, and I would like to submit that, unless you take $x$ rays on weightbearing or under load, you cannot properly estimate the degree of cartilage thickness. This is something that is not done routinely and I think should be.

MR. TAYLOR Regarding Mr. Kates' comments about the regeneration of joint synovium in 12 months, this is known to occur experimentally in as little as $\mathbf{6 0}$ days, and our arthrograms after synovectomy show that at least in the human knee joint there may be complete regeneration at 3 months. We have taken earlier arthrograms. I have one taken at 3 weeks with evidence of a supra-patellar space. Regarding the $x$-ray interpretation: I am in complete agreement. This is an exceedingly difficult problem. The radiographs vary not only with weightbearing but with the radiographer who takes them, the position of the knee, and the density of the $x$ ray; and repeat $x$ rays on the same patient will pick up further 